\title{
白色念珠菌热休克蛋白 90
}

王 丽 朱篠娟

( 东北师范大学遗传与细胞研究所, 长春 130024)

摘要综述了近 10 年来国际上关于白色念珠菌热休克蛋白 90 研究的进展情况. 白色念珠菌 是一种人和动物的常住型寄生菌. 当人体的免疫力下降时, 它会侵入深层组织和血液循环系 统, 引起浅层或系统感染。系统感染危害很大, 死亡率高达 $70 \%$. 热休克蛋白 90 是白色念珠 菌的一种免疫优势抗原, 它作为一种分子伴侣在念珠菌感染过程中扮演着重要角色. $47 \mathrm{ku}$ 多 肽是热休克蛋白 90 的降解产物, 从系统性白色念珠菌感染康复的病人血清中检测到了高效价 的抗 $47 \mathrm{ku}$ 多肽的抗体, 不过, 因此病死亡或病情恶化的病人血液中则不存在这种抗体或效价 很低. 白色念珠菌热休克蛋白 90 的 3 个常见表位已被确定, 即表位 C, B, H. 表位 C 与表位 H 具有免疫原性. 这两种表位在血清学检测中显示出了高度的特异性和灵敏性, 有可能发展成 为一种有效的白色念珠菌感染的血清检验方法. 最新实验表明: 热休克蛋白 90 是一种 ATP 依 赖型的分子伴侣, 参与细胞调控蛋白的折叠和应激变性多肽的重新折叠. 还介绍了一些关于 白色念珠菌感染的治疗和其他真菌热休克蛋白 90 研究的结果.

\section{关键词 白色念珠菌 热休克蛋白 90 抗原表位}

白色念珠菌( Candida albicans) 是一种栖生于动物和人类口腔和肠胃道的条件致病菌. 它 可以作为一种机会病原体在人体免疫力下降和正常菌群失调时, 侵入深层组织或进入血液循 环系统, 而引起浅层和系统性 (又称深层, 全身性) 感染. 口腔和食道的白色念珠菌感染是艾滋 病病人的常见临床症状, 大多数的艾滋病病人患有这种感染, 特别是儿童患者 ${ }^{[1]}$. 系统性白色 念珠菌感染危害很大, 据英国科学家统计, 在使用了抗真菌药物后, 系统白色念珠菌感染的死 亡率仍高达 $70 \%$ 以上 ${ }^{[2]}$. 令人忧心的是, 白色念珠菌对血循环的感染病例在快速增加. 在美 国，仅于 1980 1989 年的 10 年间，白色念珠菌血循环感染便增加了 $219 \%$ 4 $87 \%$, 已超过了 克雷氏杆菌, 相当于大肠杆菌感染的发病率. 系统性白色念珠菌感染得不到控制的主要原因: 一是缺乏对致病机理的了解; 二是缺乏有效的早期诊断方法和治疗途径. 有关宿主的免疫系 统如何抵抗白色念珠菌感染的机理问题, 多年来一直是科学界关注的焦点. 英国曼切斯特医 学院的 Matthews 等人 ${ }^{[3]}$ 长期以来从事白色念珠菌感染免疫学方面的研究工作, 取得了许多重 要的研究进展. 他们发现, 白色念珠菌的免疫优势抗原是热休克蛋白 90 . 在系统性感染中, 保护性抗体的一个主要靶位与人类的热休克蛋白 90 的表位相同. 他们认为, 在系统性白色念 珠菌感染中, 热休克蛋白 90 起着主要的病理学作用. 热休克蛋白 90 的研究对开发白色念珠 菌感染的免疫疗法具有重要的意义, 免疫疗法是对白色念珠菌感染进行有效治疗的最有希望 的途径之一.

近年来, 热休克蛋白 90 的研究, 特别是白色念珠菌热休克蛋白 90 的研究, 已引起国外许 多研究者的兴趣, 本文重点介绍国际上对白色念珠菌热休克蛋白 90 研究所取得的一些进展.

\section{1 热休克蛋白家族}

原核和真核细胞是通过增加一类叫热休克蛋白 (又名热激蛋白, heat shock proteins, HSPs) 
的合成速率来对付温度突然升高所产生的压力. 目前, 人们已经清楚这一类蛋白存在于正常 的细胞中, 并行使重要的生理功能. 当细胞受到外来刺激时, HSPs 会参与细胞的应激反应. 早 在 1987 年, Ellis ${ }^{[4]}$ 就发表了一篇题为“分子伴侣蛋白质” (Proteins as molecular chaperones) 的文 章. 作者用管家蛋白 (house_keeping proteins) 一词来表示这类蛋白质的一些通用功能. 现在, 人们对分子伴侣的功能有了更广泛的认识, 比如: 协助新生肽的折叠, 帮助新生肽成熟为活性 蛋白, 参与 DNA 复制和细胞的应激反应等等. 热休克蛋白家族中的大部分蛋白已被鉴定为分 子伴侣. 研究得较多的有热休克蛋白 90 , 热休克蛋白 70 , 热休克蛋白 65 和热休克蛋白 60 .

分枝杆菌的热休克蛋白 65 与人体自身反应 $\mathrm{T}$ 细胞可以同时被检测, 因此, 人们认为热休 克蛋白可能与自身免疫疾病有关系. 已经发现某些疾病与抗某类热休克蛋白的抗体增加有一 定联系, 比如: 类风湿关节炎与热休克蛋白 65 , 关节僵硬脊椎炎与热休克蛋白 90 , 系统性红斑 狼疮与热休克蛋白 70 及 90 等. 在一些由细菌、原虫和蠕虫引起的感染中, 热休克蛋白( 热休 克蛋白 $60,70,90)$ 家族的成员是免疫系统的主要靶子.

\section{2 热休克蛋白 90 与抗白色念珠菌免疫}

细胞免疫与体液免疫均参与了宿主的白色念珠菌免疫. 细胞免疫与浅层白色念珠菌感染 有关, 而体液免疫则与系统性感染有联系. 细胞介导免疫缺陷的病人, 比如: 艾滋病患者, 常常 患有浅层白色念珠菌感染却很少发现系统性感染. 而在小鼠实验中, 体液免疫则在抑制系统 性白色念珠菌感染方面发挥重要的作用 ${ }^{[2]}$. 1996 年, Walsh 等人 ${ }^{[5]}$ 的工作又表明白色念珠菌 能够刺激 $\mathrm{T}$ 淋巴细胞的选择性增加. 尽管, 白色念珠菌免疫的真正机理还缺乏一个完整和确 切的答案, 但是, Matthews 小组的工作在这个问题的研究方面有了一定的突破. 他们证明白色 念珠菌感染与白色念珠菌热休克蛋白 90 有关. 从病情好转或痊愈的病人的血清中均检测出 了一种高效价的抗白色念珠菌的 $47 \mathrm{ku}$ 多肽抗原 (热休克蛋白 90 的一种降解产物) 的抗体, 而 在感染恶化和因系统性感染死亡的病人血液中未检测到该抗体或效价很低 ${ }^{[2,6]}$. 另外, 值得 注意的是, 在慢性皮肤粘膜白色念珠菌感染和艾滋病患者中 $47 \mathrm{ku}$ 抗原产生抗体的比例很高, 而他们却很少出现深层的白色念珠菌感染. 动物实验证明, $47 \mathrm{ku}$ 抗原的抗体对深层或系统性 白色念珠菌感染具有保护作用 ${ }^{[2]}$.

\section{3 热休克蛋白 90 抗原表位图谱}

Matthews 等人利用系统性白色念珠菌感染、浅层白色念珠菌感染和从白色念珠菌感染康 复的患者血清, 确定了白色念珠菌热休克蛋白 90 的抗原表位图谱, 发现了 3 个能够与病人血 清起反应的常见表位, 即表位 C, 表位 B 和表位 H. 表位 C(LKVIRK) 能够与所有病人的血清起 反应．表位 B(LSREM) 是次常见的识别表位．表位 H (DEPAGE) 是一种白色念珠菌特有的表 位, 它可以被大约 $50 \%$ 的病人血清识别. 免疫印迹检测证明白色念珠菌热休克蛋白 90 的分子 量约为 $92 \mathrm{ku}$. 实验表明, $47 \mathrm{ku}$ 和 $40 \mathrm{ku}$ 多肽均是白色念珠菌热休克蛋白 90 的降解产物. 利 用免疫印迹方法还发现, 抗表位 C 和表位 $\mathrm{H}$ 的兔抗血清可以与热休克蛋白 90 的 $47 \mathrm{ku}$ 或 40 $\mathrm{ku}$ 多肽起免疫反应. 而表位 $\mathrm{B}$ 则不具有免疫原性，推测表位 $\mathrm{B}$ 是碳水化合物的结合位点 ${ }^{[6]}$.

人工合成的表位 $\mathrm{C}$ 和表位 $\mathrm{H}$ 与适当的载体连接后, 可以用作探针进行白色念珠菌感染的 血清学鉴定. 在斑点免疫印迹实验中, 表位 $\mathrm{C}$ 探针在灵敏性和特异性上优于未提纯的超免疫 兔抗白色念珠菌血清, 次于经亲和纯化的抗 $47 \mathrm{ku}$ 抗原的抗体. 由于亲和纯化的抗体在制备 
技术上有一定的困难, 抗原表位探针有可能成为一种白色念珠菌感染的血清学诊断方法 ${ }^{[0]}$. 利用生物技术生产的热休克蛋白 90 特异性抗体在鼠模型中显示了保护作用 ${ }^{[7]}$. 抗热休克蛋 白 90 抗原表位 C(LKVIRK) 的鼠单克隆抗体使鼠的死亡率下降了 $23 \%$ ～40\%。重组抗体的治 疗效果还表现在对肾脏感染白色念珠菌的治疗上. Matthews 等人 ${ }^{[8]}$ 认为, 这种抗体介导的保 护作用可能与在循环系统中白色念珠菌热休克蛋白 90 的结合特性产生的中和作用有关, 因为 LKVIRK 表位在体外能够牢固地与地塞米松结合.

在人类与白色念珠菌之间表位 $\mathrm{C}$ 是高度保守的. 这表明, 系统白色念珠菌感染的病人体 内产生的是抗自身表位的自身抗体. 在一个动物模型中, 具有对保守表位产生自身抗体的病 人的血清对系统白色念珠菌感染具有保护作用. 在提前注射了具有抗热休克蛋白 90 表位 C 抗体的病人血清后, 鼠的死亡率降低了 $50 \%{ }^{[9]}$.

\section{4 热休克蛋白 90 的作用机理}

热休克蛋白 90 是一种分子伴侣. 分子伴侣在发挥作用时的第一步是识别靶蛋白, 现在, 人们对这个问题的了解还不很多. 比较明确的认识是分子伴侣只识别非天然结构, 而不识别 天然的结构. 推测分子伴侣可能与蛋白的疏水表面相结合, 但在天然分子中, 疏水残基往往位 于分子的内部. 在蛋白去折叠或在新生肽链的折叠过程中, 疏水表面可能会暂时暴露, 这样分 子伴侣便可以与之相结合了. 分子伴侣是如何识别非天然结构还不清楚. 第二步是分子伴侣 与靶蛋白结合形成复合物. 不同的分子伴侣与不同的靶蛋白结合的亲和力是不同的. 不同的 分子伴侣的释放机制也不相同 ${ }^{[10]}$.

对于热休克蛋白 90 的作用机理还不很清楚, 特别在热休克蛋白 90 是否是 ATP 依赖型的 问题上存在着争论. 但是, 最近 Obermann 等人 ${ }^{[1]}$ 的工作表明, 热休克蛋白 90 是 ATP 依赖型 的分子伴侣, 参与细胞调控蛋白的折叠和应激变性多肽的重新折叠. 在生理学上. 热休克蛋 白 90 可以与许多细胞中的蛋白质相结合, 在达到适当的细胞内位置或接受到正确的活动信号 时, 才会被激活 ${ }^{[3]}$. Schwartz 和 Mizukami 认为, 热休克蛋白 90 与所结合的蛋白质之间的相互作 用依赖于热休克蛋白 90 一般结构特征, 并指出, 亮氨酸拉链 (leucine zipper), 如 M_L_L_L 区域, 有可能是热休克蛋白 90 二聚体的蛋白结合位点 ${ }^{[121}$. 对系统白色念珠菌感染有保护作用的热 休克蛋白 90 的表位 C 正处于这个蛋白结合位点中心. 这一点也许可以解释为什么表位 C 会 如此有效, 推测它能够阻止真菌热休克蛋白 90 与血清蛋白结合. 还有一种可能性, 即真菌的 热休克蛋白 90 本身在异常的细胞外环境中有着某种特异的生物学活性. 例如, 它可能影响血 凝过程. 利用抗凝血酶原 (prothrombin) 的兔抗血清已将人类热休克蛋白 90 的序列研究清 楚 ${ }^{13]}$. 热休克蛋白 90 和凝血酶原均携带一个三肽 VIR 结构, 与表位 $\mathrm{C}$ 相似, 这一结构是凝血 酶原的催化部位.

人类的热休克蛋白 90 存在于细胞内, 其功能涉及到蛋白质高级结构的组装和解体、结合 不同的细胞蛋白质和调节蛋白质的活性等. 在系统白色念珠菌感染病人的血浆中, 有许多类 似多功能热休克蛋白 90 的循环物, 它们在细胞外环境中模拟人类热休克蛋白 90 的生物学活 性. 不论人类还是白色念珠菌热休克蛋白 90 在细胞生理学上的作用机理还有待于进一步的 工作 ${ }^{[2]}$.

白色念珠菌热休克蛋白 90 的基因结构和调控已有新的进展. Swoboda 等人利用与白色念 珠菌有同源性的啤酒酵母菌 (Saccharomyces cerevisiae) 的热休克蛋白 82 探针从 cDNA 的基因文 
库中篮选分离鉴定了白色念珠菌热休克蛋白 90 的基因序列. 研究表明，热休克蛋白 90 基因 不含内含子，与其他真菌、脊椎动物和植物的热休克蛋白 90 的同源性为 $61 \% \sim 79 \%$. 利用定 量的 RNA 分析法发现, 热休克蛋白 90 是热激诱导的, 水平的高低与所处的时期有关, 高峰出 现在指数生长中期. 在从孢子型的菌株向菌丝型转变的时期, 热休克蛋白 $90 \mathrm{mRNA}$ 的水平出 现了瞬间增加的现象. Southern 印迹表明在二倍体的白色念珠菌的基因组中,仅有一个热休克 蛋白 90 的基因座位. 单一的热休克蛋白 90 基因位点是保持白色念珠菌活性所必需的 ${ }^{[14]}$.

\section{5 真菌热休克蛋白 90}

在其他一些微生物中( 例如: 酵母 Candida parapsilosis, 革兰氏阳性菌 Corynebacterium jeikeiun 和 丝状真菌Aspergillus fumigatus) 也发现了热休克蛋白 90 . 这些蛋白与有关的病原体 感染之间存在什么样的内在关系, 还不清楚. Burnie 和 Matthews 发现真菌热休克蛋白 90 在病 人体内的循环伴随着侵染性曲霉病的发生 ${ }^{[15]}$.

Saccharomyces cerevisiae 已被证明是一种能够对人类产生慢性侵害的酵母菌，尽管侵害的 机率很小. 小鼠实验表明, 这种菌的热休克蛋白 90 的过量表达会增加对鼠的肾、肝和脾等器 官产生的毒害, 致死率也随之增加. 此热休克蛋白 90 的氨基酸序列与白色念珠菌的热休克蛋 白 90 有 $84 \%$ 的同源性 ${ }^{[16]}$.

与革兰氏阴性细菌释放内毒素一样，真菌热休克蛋白 90 是在酵母降解时被释放的. 与 抗生素药物可以引起内毒素增多相似, 抗真菌药物两性霉素 $\mathrm{B}$ 可以加速酵母的裂解, 使真菌 热休克蛋白 90 沉淀增加. 热休克蛋白 90 的抗体可以缓解由两性霉素 B 引起的毒副作用. 临 床医生为了减少这种危害, 在使用两性霉素 $\mathrm{B}$ 时, 采用逐步增加剂量的方法, 以达到最佳的治 疗效果 ${ }^{[3]}$. Ziegler 等人在治疗革兰氏阳性细菌感染时, 采用抗内毒素的鼠抗人单克隆抗体和 抗菌素同时使用的方法收到了好的疗效 ${ }^{[17]}$. 另外, Driscoll 等人证明,一种存在于人类唾液中 的蛋白家族(Histatins) 中的 Hst5 具有抗白色念珠菌感染的能力. Hst5 共由 24 个氨基酸组成, 其中一个 13 个氨基酸区段单独具有杀死真菌病原体的能力. 由于去壁的白色念珠菌原生质 球也对 Hst5 敏感, 所以推测细胞壁在 Hst5 杀死念珠菌的过程中不是必要的成分 ${ }^{[18]}$. Tsai 等 人的工作表明, Hst5 对于唑类抗真菌药物有效和无效的菌株均具有同样的杀伤能力，提示 Hst5 的杀念珠菌的方式与唑类抗真菌药物有所不同 ${ }^{[19]}$.

\section{6 结束语}

从 80 年代开始至今, 经过众多科学工作者的共同努力, 人类对白色念珠菌的热休克蛋白 90 的认识在逐步加深. 最近, 又有研究表明, 热休克蛋白 90 与其他疾病的发生也有密切的关 系 ${ }^{[20,21]}$. 一些基础研究工作正在加紧进行. 美国的加利福尼亚医学研究中心、蒙大拿州立大 学、乔治城大学和内华达大学 4 个单位成立了白色念珠菌研究中心. 这里集中了一批在细胞 识别领域富有经验的研究专家, 他们将重点研究有关白色念珠菌的附着机理和其他基础理论 问题. 我们相信在不久的将来在这一领域的研究会有更多的成果问世. 另外, 国外的一些实 验室, 也在利用基础研究成果进行临床应用研究, 在利用热休克蛋白 90 抗体对白色念珠菌感 染进行治疗和生物疫苗研究等方面将有新的进展. 应该指出的是, 我国不论在白色念珠菌感 染的基础研究还是在临床应用研究方面均与国际先进水平相差较大. 在我们这样一个人口众 多, 医疗水平不高的国家, 开展对这样一种危害很大的真菌感染的研究, 更具有其现实意义. 
致谢＼cjkstart本工作为吉林省科委应用基础基金( 批准号：970556_1)资助项目．

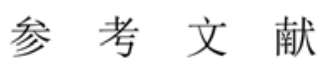

1 O' Connell B C, Xu T, Walsh T J, et al. Transfer of a gene encoding the anticandidal protein histatin 3 to salivary glands. Hum Gene Ther, 1996, 18: 2255 2261

2 Matthews R C. Candida albicans HSP 90: link between protective and auto_immunity. J Med Microbiol, 1992, 36: 367 370

3 Matthews R C, Burnie J P. The role of hsp90 in fungal infection. Immunol Today, 1992, 13( 9) : 345 348

4 Ellis J. Proteins as molecular chaperones. Nature, 1987, 328: 378 379

5 Walsh P, Norris D A, Abe J. Candida albicans induces selective expansion of human T lymphocytes expressing the T_cell receptor variable region V beta 5. 1. J Dermatol Sci, 1996, 12(2) : 140 146

6 Matthews R, Burnie J P, Lee W. The application of epitope mapping in the development of a new serological test for systemic candidosis. J Immunol Methods, 1991, 143: 73 79

7 Matthews R, Burnie J. Antibodies against Candida: potential therapentics? Trends Microbiol, 1996, 4( 9) : 354 358

8 Matthews R, Hodgetts S, Burnie J. Preliminary assessment of a human recombinant antibody fragment to hsp90 in murine invasive candidasis. J Infect Dis, 1995, 171(6) : 1668 1671

9 Matthews R C, Burnie J P, Howat D, et al. Autoantibody to heat shock protein 90 can mediate protection against systemic candidosis. Immunology, 1991, 74: 20 24

10 王志珍. 新生肽链的折叠与分子伴侣. 见: 李宝健主编. 面向 21 世纪生命科学发展前沿. 广州: 广东科技出版社, 1996, 93 105

11 Obermann W M J, Sondermann H, Russo A A, et al. In vivo function of hsp90 is dependent on ATP binding and ATP hydrolysis. J Cell Biol, 1998, 143(4) : 901 910

12 Schwartz J A, Mizukami H. A metal_linked gapped zipper model is proposed for the 90KUa heat shock protein_estrogen receptor interface. Med Hypoth, 1991, 35: 140 145

13 Rebbe N F, Ware J, Bertina R M, et al. Nucleotide sequence of a cDNA for a member of the human 90_kua heat_shock protein family. Gene, 1987, 53: 235 245

14 Swoboda R K, Bertram G, Budge S, et al. Structure and regulation of the HSP90 gene from the pathogenic fungus Candida albicans. Infect Immun, 1995, 63(11): 4506 4514

15 Burnie J P, Matthews R C. Heat shock protein 88 and Aspergillus infection. J Clin Microbiol, 1991, 29( 10) : 2099 2106

16 Hodgetts S, Matthews R, Morrissey G, et al. Over_expression of Saccharomyces cerevisiae hsp90 enhances the virulence of this yeast in mice. FEMS Immunol Med Microbiol, 1996, 16(3_4) : 229 234

17 Ziegler E J, Fisher C J, Sorung C L, et al. Treatment of gram_negative bacteria and septic shock with HA_IA human monoclonal antibody against endotoxin. New Engl J Med, 1991, 324: 429 436

18 Driscoll J, Duan C, Zuo Y, et al. Candidacidal activity of human salivary histatin recombinant variants produced by site_directed mutagenesis. Gene, 1996, 177( 1): 29 34

19 Tsai H, Bobek L A. Studies of the mechanism of human salivary histatin_5 candidacidal activity with histatin_5 variants and azole_sensitive and 3/resistant Candida species. Antimicrob Agents Chemother, 1997, 41(10): 2224 2228

20 Galea_Lauri J, Richardson A J, Latchman D S, et al. Increased heat shock protein 90 ( hsp90) expression leads to increased apoptosis in the monoblastoid cell line U937 following induction with TNF_alpha and cycloheximide: a possible role in immunopathology. J Immunol, 1996, 157(9) : 4109 4118

21 Stahl M, Ludwig D, Fellermann K, et al. Intestinal expression of human heat shock protein 90 in patients with Crohn's disease and ulcerative colitis. Dig Dis Sci, 1998, 43(5) : 1079 1087 